\title{
sLORETA neurofeedback in fibromyalgia
}

\author{
Rubén Pérez-Elvira* and Ana Jiménez Gómez
}

Psychophysiology Lab (NEPSA Rehabilitación Neurológica), Salamanca, Spain.

* Correspondence: neuropsicologia@nepsa.es; Tel.: +34 923057037

Received: 5 November 2019; Accepted: 14 January 2020; Published: 30 January 2020

Edited by: King-Hwa Ling (Universiti Putra Malaysia, Malaysia/Harvard Medical School, Boston, USA)

Reviewed by: Garry Kuan (Universiti Sains Malaysia, Malaysia); Amedeo D’Angiulli (Carleton University, Canada) https://doi.org/10.31117/neuroscirn.v3i1.40

\begin{abstract}
Fibromyalgia is a chronic and incapacitating condition that produces, as main symptoms, pain, and stiffness. In addition to these physical symptoms, it is also accompanied by psychological symptoms such as cognitive deficits, anxiety, and depression. One of the non-pharmacological treatments that have been used in this pathology in recent years is neurofeedback. In this study, we analyze the efficacy of sLORETA Neurofeedback in the case of fibromyalgia. The experimental subject was a 37-year-old patient. Quantified electroencephalography studies were applied on three occasions, one initial, another after fifteen days of a waiting list, and another after treatment. Psychometric scales were also applied at the same time to evaluate the patient's psychological and physical state. The treatment consisted of 5 sessions of Neurofeedback LORETA in Brodmann area 2. After the treatment, a neurometric, psychometric, and clinical improvement were found. The improvement of the patient after 5 sessions is relevant since previous studies using neurofeedback in fibromyalgia, despite positive results, suggest a higher number of sessions were needed to achieve stronger results. Therefore, the intervention with Neurofeedback LORETA in fibromyalgia patients could be an alternative or complement to current treatments.
\end{abstract}

Keywords: Neurofeedback; sLORETA; Fibromyalgia; QEEG

C2020 by Pérez-Elvira and Jiménez Gómez for use and distribution in accord with the Creative Commons Attribution (CC BY-NC 4.0) license (https://creativecommons.org/licenses/by-nc/4.0/), which permits unrestricted non-commercial use, distribution, and reproduction in any medium, provided the original author and source are credited.

\subsection{INTRODUCTION}

Fibromyalgia (FM) is a chronic disease that causes muscle pain and fatigue, whose main symptoms are pain and tenderness at various points of the body, usually located in the neck and shoulders, back, hip, and extremities (arms and legs). Usually, the person suffering FM has other chronic conditions such as irritable bowel syndrome, chronic fatigue syndrome, interstitial cystitis, among others. This disorder can appear in anyone, but is more common in women and usually begins in middle age. Certain diseases such as rheumatoid arthritis or lupus may make the onset of FM 
more likely (NIH: Instituto Nacional de Artritis y Enfermedades Musculoesqueléticas y de la Piel., 2018). In addition, symptoms such as muscle stiffness, insomnia, headache, menstrual cramps, and cognitive problems may also exist. These patients tend to have memory and attention impairments; neuropsychological tests reflect dysfunctions, mainly in long-term memory, verbal fluency and information processing speed (Alanoğlu et al., 2005; Howe \& Sterman, 1972; Ozgocmen et al., 2003).

According to Donaldson and colleagues (Donaldson et al. 1998), this is the difference between FM and other similar conditions: myofascial syndrome (MF). Both conditions are similar, but the main distinguishing factor is the psychological profile. Patients with MF do not present psychological dysfunctions in relation to the general population, however, in FM they show high values of stress, anxiety, and depression, in addition to notable cognitive deficits; a correlation has been found between the severity of the pain in patients with FM and the psychological dysfunctions. In other words, these authors suggest that FM differs from MF in the central nervous system (CNS) involvement in the former.

One of the leading hypotheses about chronic pain in FM, and it would be due to a lowering of the threshold of pain receptors or a higher excitation capacity of CNS neurons involved in pain transmission and perception. This sensory disturbance can affect sleep, mood and impair neurotransmitter and hormonal balance (Mueller et al., 2001). Some authors have found the primary somatosensory cortex (Brodmann Areas 1, 2 and 3 ) to be involved in pain processing (Kanda et al., 2000) and have related not only somatic dysfunctions but also non-somatic dysfunctions in FM with this cortex (Kim et al., 2015). Currently, most treatments are focused on symptom management. In this sense, cognitive therapy has shown to be very helpful for patients who suffer FM since they are prone to adopt non-adaptative behaviors, hopelessness and/or depression (National Fibromyalgia Association, 2018). Despite the availability of a great diversity of treatments (pharmacological therapy, aerobic exercise, biological recovery, physical therapies, and multidisciplinary therapies), their effectiveness is moderately successful, and most of the patients are still very impaired and, sometimes, disabled (Mueller et al., 2001).

Neurofeedback (NF) is a specialized field of biofeedback focused on the electroencephalographic (EEG) activity control (Carrobles, 2016). It is based on operant conditioning (i.e., behavior modification through reward and punishment) applications to EEG activity. In recent years, only a few studies have been carried out on the effect of Neurofeedback on FM. Kayiran and colleagues (Kayiran et al., 2007) applied an NF protocol to three FM patients. The protocol consisted of reducing the theta/beta waves ratio. The symptoms and clinical conditions of these patients were evaluated before and after treatment with a visual analog scale of pain and fatigue, Hamilton anxiety and depression scale, Beck depression and anxiety inventory, and Short Form-36 Health Survey (SF-36). After ten sessions, the intervention alleviated most of the symptoms with the observation of post-treatment improvement on all scales (Hamilton Anxiety Scale, Hamilton Depression Scale, Beck Depression Inventory, Beck Anxiety Inventory and SF-36).

In Donaldson's study (Donaldson et al., 1998) involving 252 subjects with $\mathrm{FM}$ and MF, an anomalous dominant frequency was found in EEG analyses in 13 brain regions, where only successful results of fibromyalgia symptoms were obtained after a combined program that included treatment with NF. There were also changes in the EEG pattern in those patients with FM who manifested cognitive problems, leading to the improvement of the rest of the presentations.

Muller conducted a study (Mueller et al., 2001) with 30 subjects, which demonstrated significant improvements in the symptomatology associated with $\mathrm{FM}$ and changed EEG patterns after the application of EEG-directed stimulation treatment. They also showed improvements in cognitive processing, mood, sleep, and fatigue. Almost $50 \%$ of the subjects returned to work and thus abandoned their state of disability. After an 8- 
month follow-up from the end of treatment, the benefits were maintained over time. Despite the application of other therapies, the main significant differences were primarily explained based on the first phase of treatment. They concluded that EEG training was the main component of therapeutic efficacy.

Kayıran and colleagues (Kayıran et al., 2010) conducted another randomized study of 36 subjects with FM. The design was randomized and blinded, where 18 patients received twenty sessions of the sensorimotor rhythm (SMR) NF treatment for 4 weeks, and 18 patients (control group) received $10 \mathrm{mg} /$ day of escitalopram for 8 weeks. Psychometric tests were administered (visual analog scales of pain and fatigue, Hamilton and Beck's anxiety and depression inventory, fibromyalgia impact questionnaire, and SF-36) before, during and after treatment. In both groups, there were significant improvements in post-treatment measurements, with greater benefits for the NF group in all parameters. This study showed the efficacy of NF for the treatment of pain and psychological symptoms and for improving the resulting quality of life of FM.

As mentioned above, at present, few studies exist regarding the application of NF in FM, and they are based on amplitude and single-channel neurofeedback (Donaldson et al., 1998; Kayiran et al., 2007; Kayıran et al., 2010; Minhoto et al., 1997; Schwartz, 1995). The technology in the field of neurotherapy has developed enormously in recent years, and we now have better measurement and intervention instruments and more advanced procedures (Hammer et al., 2011). One of these new procedures consists of using triangulation by inverse solution methods of the EEG signal sources (LORETA, VARETA, etc.) when applying the intervention with neurofeedback.

Brain Master Standardized Low-Resolution Electromagnetic Tomography NF (sLORETA) allows the source of EEG activity within the cortex to be triangulated. Its bark model is made up of 6,239 voxels, cubes of 5 cubic millimeters. In each voxel, the current density source (CDS) is $1-45 \mathrm{~Hz}$, whose estimates can be converted into a three-dimensional image showing changes in real-time (Gracefire, 2016). Each structure is formed by a set of voxels (Figure 1 ) that are considered or designated as Regions of Interest (ROI) and displayed for monitoring through the screen (Collura, 2012; Collura, 2017). Feedback, in the case of sLORETA amplitude interventions, is provided when the selected ROI produces an amplitude in the chosen band that is above or below, depending on whether we try to reinforce or inhibit the threshold that the clinician sets up (Gracefire, 2016). The choice of ROls in which to increase or decrease the amplitude is made by crossing patient symptom data, the functionality of these ROls and their deviation or not from the norm (Smith, 2017). sLORETA allows designing protocols aimed at more substantial or smaller cortical areas, including individual voxels or predetermined sets of them. This approach, due to its hyper specificity, can produce rapid changes in brain activity (Collura, 2012). This study aims to analyze the efficacy of sLORETA guided in the treatment of FM psychological symptoms.
(A)

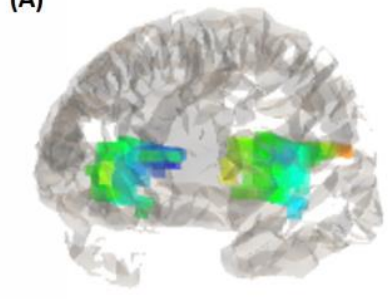

(B)

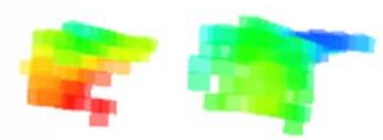

Figure 1. The image was taken from the sLORETA projector of BrainAvatar. (a) View of the ROI (insula) integrated into a representation of the brain. (b) View of the voxels that compose this ROI.

\subsection{MATERIALS AND METHODS}

\subsection{Subject}

The patient was a 37-year-old woman diagnosed with FM from the age of 24 by a competent rheumatologist. She also associated anxious and depressive symptoms. So far, she had received psychological therapy and physiotherapeutic intervention. At the time of starting this study, she was not receiving any intervention. 
At the moment of the evaluation, the patient was suffering from intense generalized pain on both sides of the body, although with a predominance of the left side and severe anxiety. Besides, she was suffering from a depressed mood as a result of her state of health.

During our intervention, the patient did not take any medication.

\subsection{Procedure}

We obtained written informed consent from the patient to run our single case study. The study was conducted within NEPSA Neurological Rehabilitation, a clinic licensed by the Department of Health Management of the Junta de Castilla y León as a multidisciplinary care center for neurological patients and was carried out as a treatment of the patient, so no approval of an ethics committee was necessary.

The EEG was registered for later quantification, with eyes open and closed in resting condition. A waiting list of fifteen days was applied, after which, before the start of treatment, a new EEG was registered, which was also quantified, and evaluations of the state of mental health and pain were carried out using psychometric instruments both before and after treatment.

An amplitude sLORETA protocol was administered to the patient, with a total of 5 sessions, with a treatment duration of 15 days, after which a new EEG was collected and analyzed.

\subsection{Psychometric instruments}

\section{Beck Anxiety Inventory (BAI)}

Beck Anxiety Inventory (BAI) (Beck et al., 1988) was administered in a Spanish adaptation (Sanz, 2019). BAI is made up of a list of anxiety symptoms in which the patient indicates whether or not the symptom is present and its severity on a Likert scale.

\section{Beck Depression Inventory-II (BDI-II)}

The Spanish version (Derogatis \& Unger, 2010) of the Beck Depression Inventory-II (DAI-II) (Beck et al., 1996) was used to record the presence and severity of depressive symptoms. It is a self-administered inventory of depression symptoms.

\section{Symptom Checklist 90-R (SCL-90-R)}

This Inventory (Derogatis \& Unger, 2010) evaluates several psychopathological characteristics in both clinical and healthy populations, and serves as a screening or to assess changes in symptoms due to treatment. It is made up of 90 items with a 5-point response (from 0 to 4 ) on a Likert type scale. Its administration takes about 15 minutes and applies to people between 13 and 65 years (INFOCOP: Consejo General de la Psicología de España, 2006).

It is divided into 9 primary dimensions: somatization (SOM), obsessions and compulsions (OBS), interpersonal sensitivity (SI), depression (DEP), anxiety (ANS), hostility (HOS), phobic anxiety (FOB), paranoid ideation (PAR) and psychoticism (PSIC); and 3 global indices of psychological distress: global index of severity (IGS), positive index of distress (PSDI) and total positive symptoms (TP).

The values assigned to each item are summed and divided by the number of completed items for each of the 9 dimensions, converting the direct scores into mean scores (T-scores). When the $\mathrm{T}$ score is greater than or equal to 65 , it indicates that the person is at risk, and a $\mathrm{T}$ score equal to or greater than 80 indicates severe pathology (Casullo, 2004).

\section{Analog Visual Scale}

Before starting the NF intervention, the patient scored her pain on a subjective analog visual scale. This scale consisted of a $10 \mathrm{~cm}$ line regulated from 0 to 100 , meaning 0 that there is no pain and 100 the worst possible pain.

\subsection{EEG recording and quantification}

EEG recordings and NFB sessions were conducted with a Discovery 20 amplifier (BrainMaster Technologies) and BrainAvatar software (BrainMaster Technologies). To record the EEG and to run the sessions, the equipment mentioned above and electrocap (Electro- 
cap International), commonly used in electroencephalography, with 19-channels according to the International 10-20 System with Linked Ears montage (Fp1, Fp2, F7, F3, Fz, F4, F8, T3, C3, Cz, C4, T4, T5, P3, Pz, P4, T6, O1, and O2) and earthing on the front of the skull were used. Impedances were kept below $5 \mathrm{k} \Omega$.

The EEGs were edited and analyzed using NeuroGuide 2.9.1 and LORETA (Key Institute) software as well as BrainAvatar Analyzer (BrainMaster Technologies) and QEEGPro normative database. The duration of the recordings was 3 minutes in each condition (open/closed eyes). EEG evaluation sessions were held at the same time of day, around 11:00 a.m.

The records were visually inspected and the artifacts (non-brain activity collected in the EEG) were removed and the EEG was quantified. The most deviated from the norm ROI was found by exporting the $Z$ scores obtained from the patient in NeuroGuide 2.9.1 to LORETA. After this, a new analysis of SLORETA was carried out with the QEEGPro database, where it was determined the ZScore value for every most deviated ROI voxels (in this case Brodmann Area 2). We also calculated the Z-Score values for every voxel in Brodmann Area 5 (BA5).

\section{5 sLORETA neurofeedback training}

The patient received five 30 minutes sessions (two sessions per week). The sessions were held at the same time of day.

The NF sessions consisted of sLORETA amplitude training in Brodmann area 2 (BA2), in this case, it was aimed at inhibiting the amplitude of the Theta band. Thresholds were set under which the Theta amplitude had to be placed to receive reinforcement and was adjusted during the sessions to ensure that the patient received reinforcement at least $50 \%$ of the time. Auditory signals were used as feedback to tell the patient when she was getting her Theta activity below the set threshold.

\subsection{Statistical Analysis}

To analyze the change in EEG values, we used an Item Analysis Approach, where ROls were assumed to be independent random variables (Bedny et al., 2007) and the voxels of these ROIs (BA2 and BA5) were treated as the "subjects" and Z scores were obtained for each voxel for the Theta frequency band, with a total of 91 voxels for BA2 (Table 1) and 75 voxels for BA5. These Zscores were dichotomized as within or outside the norm. The number of Z-scores within or outside the limits of normality was calculated after each measurement (pre-treatment1, pre-treatment2 posttreatment). Table 1 shows these scores. The McNemar's test was used to compare the percentage of Z-scores within the normal range from Theta in pre-treatment1 to Theta in pre-treatment2, and from Theta in pretreatment2 to Theta in post-treatment. These comparations were done also for BA5 (74 voxels) using McNemar's Test.

The percentages of change in the scores of the scales and inventories, as well as the change in the qualitative label of these instruments, were used to analyze clinical change. For these psychometric tests, no statistical analysis could be run because of $\mathrm{N}=1$ in these measures.

Table 1. Theta band Z scores for each voxel.

\begin{tabular}{cccc}
\hline $\begin{array}{c}\text { Voxel } \\
\text { Number }\end{array}$ & $\begin{array}{c}\text { Theta.ZAP } \\
\text { Pre1 }\end{array}$ & $\begin{array}{c}\text { Theta.ZAP } \\
\text { Pre2 }\end{array}$ & $\begin{array}{c}\text { Theta.ZAP } \\
\text { post }\end{array}$ \\
\hline 3668,00 & 2,60 & 2,51 & 1,04 \\
3683,00 & 2,51 & 2,46 & 0,98 \\
3695,00 & 2,47 & 2,43 & 0,77 \\
3711,00 & 2,28 & 2,27 & 0,58 \\
3902,00 & 5,45 & 5,32 & 0,79 \\
3917,00 & 5,89 & 5,75 & 1,09 \\
3929,00 & 5,25 & 5,15 & 1,60 \\
3930,00 & 5,98 & 5,84 & 1,24 \\
3942,00 & 5,51 & 5,40 & 1,63 \\
3960,00 & 2,66 & 2,61 & 1,07 \\
3968,00 & 2,62 & 2,58 & 1,03 \\
3979,00 & 2,59 & 2,56 & 0,98 \\
3980,00 & 2,68 & 2,63 & 0,93 \\
\hline & & Continued on next page
\end{tabular}




\begin{tabular}{|c|c|c|c|}
\hline 3989,00 & 2,46 & 2,44 & 1,02 \\
\hline 3990,00 & 2,74 & 2,69 & 0,80 \\
\hline 4001,00 & 2,70 & 2,65 & 0,72 \\
\hline 4014,00 & 2,66 & 2,60 & 0,63 \\
\hline 4218,00 & 5,30 & 5,18 & 0,90 \\
\hline 4231,00 & 5,55 & 5,43 & 1,05 \\
\hline 4243,00 & 5,14 & 5,04 & 1,51 \\
\hline 4244,00 & 5,72 & 5,59 & 1,21 \\
\hline 4253,00 & 5,23 & 5,13 & 1,61 \\
\hline 4254,00 & 5,43 & 5,32 & 1,53 \\
\hline 4255,00 & 5,63 & 5,51 & 1,44 \\
\hline 4268,00 & 5,47 & 5,36 & 1,62 \\
\hline 4278,00 & 5,48 & 5,37 & 1,70 \\
\hline 4285,00 & 2,67 & 2,64 & 1,07 \\
\hline 4295,00 & 2,68 & 2,65 & 1,04 \\
\hline 4305,00 & 2,78 & 2,72 & 0,89 \\
\hline 4320,00 & 2,64 & 2,60 & 0,93 \\
\hline 4339,00 & 2,60 & 2,56 & 0,87 \\
\hline 4358,00 & 2,55 & 2,51 & 0,80 \\
\hline 4548,00 & 5,06 & 4,94 & 0,63 \\
\hline 4565,00 & 4,89 & 4,79 & 1,18 \\
\hline 4582,00 & 5,08 & 4,98 & 1,43 \\
\hline 4598,00 & 5,40 & 5,29 & 1,31 \\
\hline 4613,00 & 5,34 & 5,23 & 1,54 \\
\hline 4625,00 & 5,41 & 5,30 & 1,63 \\
\hline 4638,00 & 2,82 & 2,78 & 1,06 \\
\hline 4646,00 & 2,86 & 2,81 & 1,01 \\
\hline 4647,00 & 2,96 & 2,90 & 0,92 \\
\hline 4659,00 & 2,99 & 2,92 & 0,86 \\
\hline 4672,00 & 2,86 & 2,81 & 0,91 \\
\hline 4673,00 & 2,99 & 2,92 & 0,80 \\
\hline 4703,00 & 2,81 & 2,75 & 0,77 \\
\hline 4936,00 & 4,66 & 4,57 & 0,96 \\
\hline 4970,00 & 5,10 & 5,00 & 1,22 \\
\hline 4971,00 & 5,20 & 5,09 & 1,09 \\
\hline 4986,00 & 5,37 & 5,26 & 1,22 \\
\hline 5000,00 & 5,36 & 5,25 & 1,45 \\
\hline 5001,00 & 5,50 & 5,39 & 1,35 \\
\hline 5011,00 & 5,44 & 5,33 & 1,55 \\
\hline 5020,00 & 3,02 & 2,96 & 0,99 \\
\hline 5021,00 & 3,14 & 3,06 & 0,89 \\
\hline 5031,00 & 3,17 & 3,08 & 0,83 \\
\hline 5043,00 & 3,05 & 2,98 & 0,88 \\
\hline 5044,00 & 3,17 & 3,08 & 0,76 \\
\hline 5333,00 & 5,11 & 5,00 & 1,00 \\
\hline 5345,00 & 5,27 & 5,16 & 1,12 \\
\hline 5355,00 & 5,31 & 5,20 & 1,36 \\
\hline
\end{tabular}

\begin{tabular}{|c|c|c|c|}
\hline 5356,00 & 5,41 & 5,30 & 1,25 \\
\hline 5362,00 & 5,41 & 5,30 & 1,47 \\
\hline 5363,00 & 2,87 & 2,85 & 1,15 \\
\hline 5369,00 & 3,20 & 3,12 & 0,90 \\
\hline 5377,00 & 3,04 & 2,99 & 0,97 \\
\hline 5378,00 & 3,29 & 3,19 & 0,73 \\
\hline 5651,00 & 4,94 & 4,84 & 1,21 \\
\hline 5652,00 & 4,99 & 4,88 & 1,06 \\
\hline 5665,00 & 5,11 & 5,00 & 1,16 \\
\hline 5672,00 & 5,23 & 5,13 & 1,28 \\
\hline 5673,00 & 5,23 & 5,12 & 1,11 \\
\hline 5676,00 & 3,31 & 3,21 & 0,84 \\
\hline 5680,00 & 3,17 & 3,10 & 0,92 \\
\hline 5689,00 & 2,92 & 2,89 & 0,99 \\
\hline 5900,00 & 4,66 & 4,58 & 1,19 \\
\hline 5911,00 & 4,89 & 4,79 & 1,14 \\
\hline 5918,00 & 5,00 & 4,89 & 1,07 \\
\hline 5924,00 & 3,07 & 3,01 & 0,94 \\
\hline 5925,00 & 3,25 & 3,16 & 0,81 \\
\hline 5932,00 & 3,04 & 2,98 & 0,85 \\
\hline 5942,00 & 3,00 & 2,93 & 0,75 \\
\hline 5956,00 & 2,97 & 2,89 & 0,67 \\
\hline 6074,00 & 3,62 & 3,54 & 0,77 \\
\hline 6084,00 & 3,97 & 3,90 & 0,88 \\
\hline 6096,00 & 4,34 & 4,26 & 1,01 \\
\hline 6105,00 & 4,62 & 4,53 & 1,12 \\
\hline 6119,00 & 3,08 & 3,00 & 0,72 \\
\hline 6126,00 & 3,04 & 2,95 & 0,66 \\
\hline 6224,00 & 3,60 & 3,53 & 0,74 \\
\hline 6232,00 & 3,92 & 3,84 & 0,84 \\
\hline 6237,00 & 4,39 & 4,29 & 0,83 \\
\hline
\end{tabular}

\subsection{RESULTS}

The pre-treatment LORETA Analysis 1 showed that the structure furthest from the norm was the $B A 2$ in the Theta band. The BrainAvatar SLORETA projector was used to locate the voxels that make up the BA2 in this projector (Figure 2). Z-scores were calculated for each of the 91 voxels that make up this structure in BrainAvatar sLORETA using the QEEGPro normative database (Table 1). The 91 voxels showed $Z$ scores higher than 1 (Table 1), $43.3 \%$ of voxels in BA5 showed 
Z-scores higher than 1 for Theta band. In the analysis after the waiting list period (pre-treatment 2), 100\% voxels in BA2 (Table 1) and $41.9 \%$ in BA5 obtained Z scores outside the normal range.

There were no significant changes $(p=1)$ between the Theta Z-scores of the 91 voxels of BA2 pre-treatment1 and pre-treatment2. The psychometric tests did not show a relevant change either (Figure 3). However, it was found that $49.45 \%$ of $Z$ scores had moved to the normal range from pre-treatment2 to post-treatment measurement (Table 3), which is a statistically significant change $(p<0.001)$. It was found $55.40 \%$ of $Z$ scores within the normal range in BA5 after post- treatment, but no significant changes in BA5 in pretreatment2 $(p=1)$ nor in the post-treatment $(p=0.754)$.

In addition, there was a pre-treatment2-post-treatment change of $81.01 \%$ in the visual analog pain scale, $20.27 \%$ in the General Severity Index of the SCL-90R, $55.26 \%$ in the BDI-II and $33.33 \%$ in the BAI scores (Figure 4).

Since there were some changes in the scores of these instruments between pre1 and pre2, the difference between the percentage of pre-treatment2-posttreatment and pre-treatment1-pre-treatment2 change was calculated in order to subtract from the final result the possible spontaneous change that had already
(A)

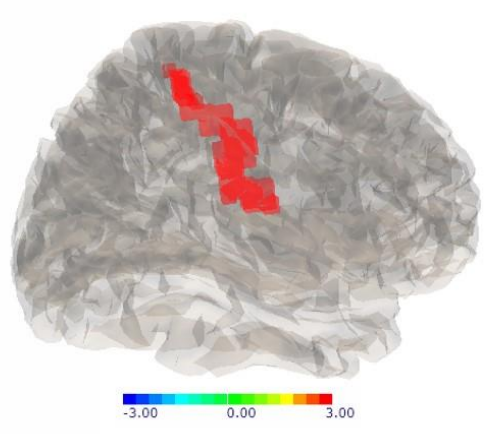

(B)

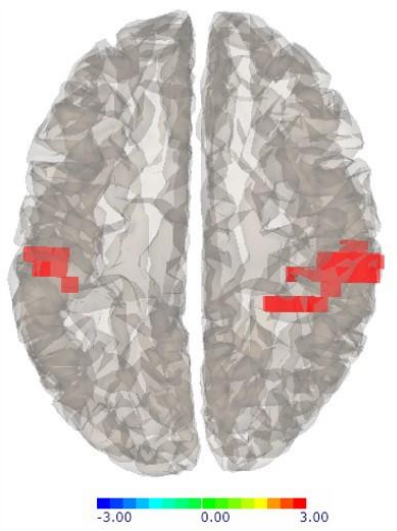

(C)

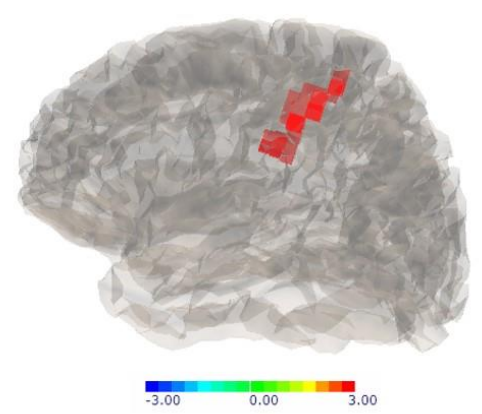

Figure 2. Brodmann Area 2. The figures show different views of Area BA2, as shown by the BarinAvatar software (in this case, it is only a sample and the colored scale has no meaning). (a) Right sagittal view, (b) axial view, and (c) Left sagittal of Brodmann Area 2.

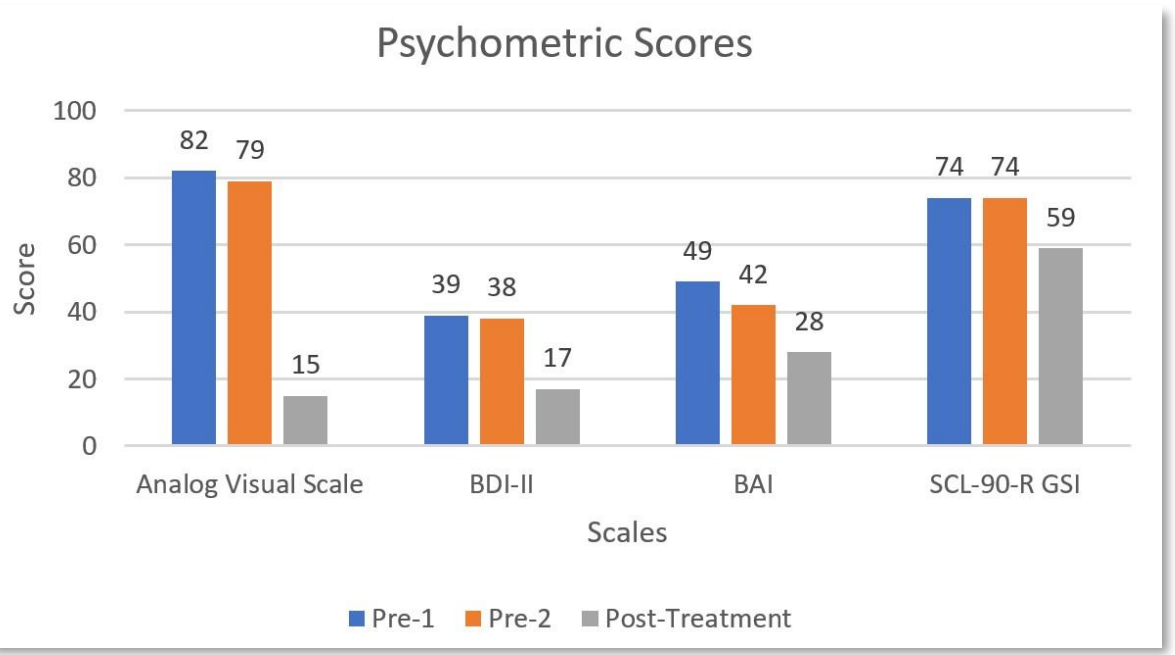

Figure 3. Psychometric scores obtained in Pre-treatment 1, Pretreatment 2, and Post-treatment. 


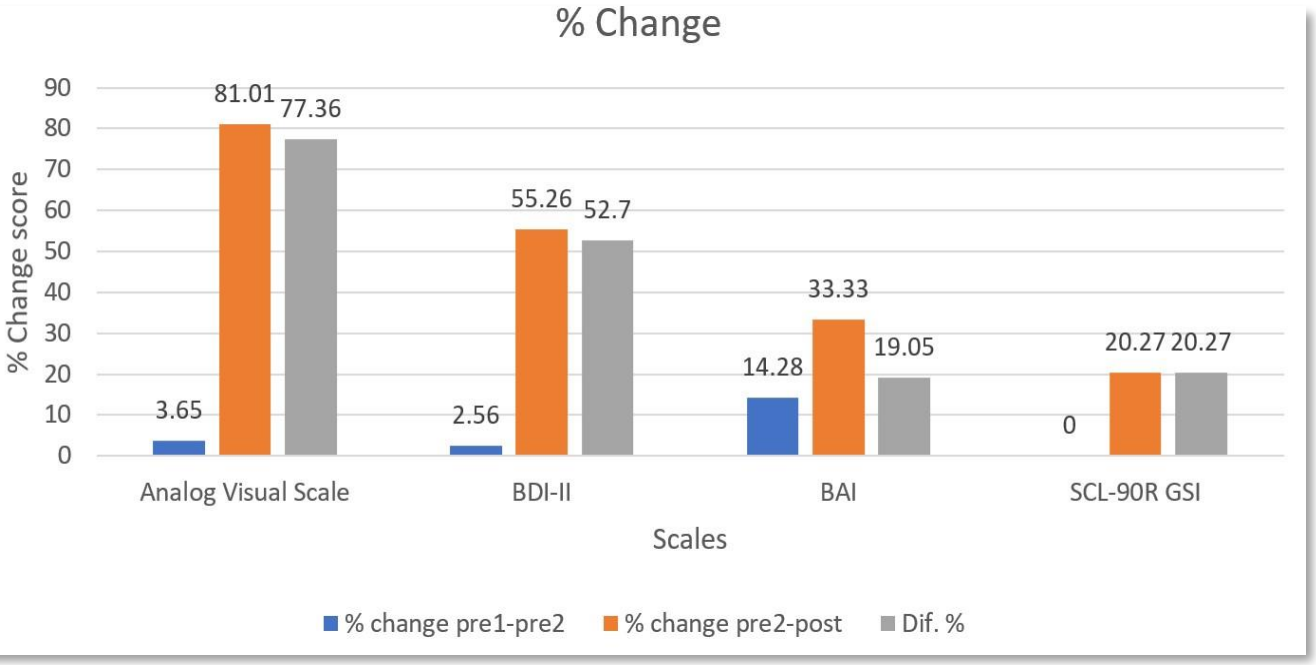

Figure 4. Percentage change pre-treatment 1-2; percentage change pre-treatment 2-post treatment; and percentage difference change pre-treatment 1-2 and pre-treatment 2-post treatment.

occurred during the waiting list condition. When a more conservative criterion was used with the scores, improvements were observed in the percentages of change of $77.36 \%$ in the visual analog pain scale, $52.7 \%$ in depression (BDI-II), 19.05\% in anxiety (BAI) and 20.27\% in Associated Psychopathology (SCL-90R) (Figure 4).

\subsection{DISCUSSION}

This study aimed to analyze the efficacy of amplitude sLORETA training in the case of FM. Some studies (Kayiran et al., 2007; Kayıran et al., 2010) had already shown results in this pathology. In comparison to these studies, we obtained results in a smaller number of sessions, probably because QEEG-guided intervention and the amplitude sLORETA NF was used instead of the surface amplitude. This type of NF is characterized by the ability to locate and train the activity of specific brain structures or even parts of them (Collura, 2014; Gracefire, 2016). In our study, the BA2 area was selected because it was the area that was most deviated from the norm, both in the pre-treatment1 and pretreatment 2 evaluation, and because of its role in the perception and anticipation of pain and body sensations (Chen et al., 2006; Kanda et al., 2000; Kim et al., 2015).

In contrast to the previous studies (Kayiran et al., 2007; Kayıran et al., 2010) that required 10 and 20 sessions respectively, in our case, the intervention protocol was reduced to 5 sessions suggesting that hyperspecific, by using sLORETA, and QEEG-guided intervention is faster than the intervention of surface NF. Our study showed that intervention with sLORETA NF produced significant changes in BA2 activity and that these changes were superior to the changes that can occur spontaneously in QEEG patterns. We controlled the spontaneous changes in BA5, which showed around $55 \%$ of Z-scores within the normal range across all evaluations and did not show any significant changes. In addition to the neurometric changes, our patient obtained relevant changes in her clinical presentation. The following very relevant improvements were observed: a decrease in pain perception of $81.01 \%$, a decrease in depressive symptoms of $55.26 \%$, a decrease in the anxiety of $33.3 \%$, and a decrease in the general severity of her symptoms of associated psychopathology of $20.27 \%$. A possible explanation for the reduction of psychological symptoms, such as anxiety or depression, could be related to the fact of the reduction of perceived pain, which could act as a mood modulator.

This study aimed to analyze the efficacy of sLORETA neurofeedback in the case of fibromyalgia. In this case, the efficacy was demonstrated not only in the improvement of the psychological complaints but also of the physical ones. Despite the results, our study had several limitations; the main ones were sample size $(\mathrm{N}=1)$ and using a visual analog scale for assessing the pain. On the other hand, the possible effect of the type of feedback used was not controlled, nor was the 
reinforcing value of the feedback evaluated. Some authors (Fisher et al., 1992; Soutar \& Longo, 2011) have indicated that the selection of the enhancer is crucial to optimize the results of NF interventions. Using ROI voxels reconstructed based on 19 electrodes was another limitation, but some authors indicated that an imperfect spatial sampling did not preclude the source of localization (Michel \& Brunet, 2019). In addition, the possible contribution of the placebo effect could not be ruled out.
Acknowledgments: No funds were received to conduct this study. We thank Dr. Javier Oltra-Cucarella for his suggestions and comments.

Author Contributions: RPE conceived, designed, performed the treatment, and analyzed the data; AJ contributed to reviewing literature; RPE and AJ wrote the paper.

Conflicts of Interest: The authors declare no conflict of interest.

\section{References}

Alanoğlu, E., Ulaş, U. H., Özdağ, F., Odabaşı, Z., Çakçı, A., \& Vural, O. (2005). Auditory event-related brain potentials in fibromyalgia syndrome. Rheumatology International, 25(5), 345-349. https://doi.org/10.1007/s00296-004-0443-3

Beck, A. T., Epstein, N., Brown, G., \& Steer, R. A. (1988). An inventory for measuring clinical anxiety: Psychometric properties. Journal of Consulting and Clinical Psychology, 56(6), 893-897. https://doi.org/10.1037/0022-006X.56.6.893

Beck, A. T., Steer, R. A., \& Brown, G. K. (1996). BDI-II, Beck Depression Inventory: Manual. Psychological Corporation.

Bedny, M., Aguirre, G. K., \& Thompson-Schill, S. L. (2007). Item analysis in functional magnetic resonance imaging. Neurolmage, 35(3), 1093-1102. https://doi.org/10.1016/j.neuroimage.2007.01.039

Carrobles, J. A. (2016). Bio/neurofeedback. Clínica y Salud, 27(3), 125-131. https://doi.org/10.1016/i.clysa.2016.09.003

Casullo, M. M. (2004). EL INVENTARIO DE SÍNTOMAS SCL-90-R de L. 8. https://www.fundacionforo.com/pdfs/inventariodesintomas.pdf

Chen, Y., Liu, H.-C., Jin, Z., Liu, G., \& Zhang, Q.-S. (2006). Functional activity mapping during anticipation of dental pain. West China Journal of Stomatology, 24(2), 121-124.

Collura, T. (2012). BrainAvatar: Integrated Brain Imaging, Neurofeedback, and Reference Database System. NeuroConnections, Summer, 31-36.

Collura, T. (2014). Technical foundations of neurofeedback. Routledge, Taylor \& Francis Group.

Collura, T. F. (2017). Quantitative EEG and Live Z-Score Neurofeedback-Current Clinical and Scientific Context. Biofeedback, 45(2), 25-29. https://doi.org/10.5298/1081-5937-45.1.07

Derogatis, L. R., \& Unger, R. (2010). Symptom Checklist-90-Revised. In I. B. Weiner \& W. E. Craighead (Eds.), The Corsini Encyclopedia of Psychology. John Wiley \& Sons, Inc. https://doi.org/10.1002/9780470479216.corpsy0970

Donaldson, C. C. S., Sella, G. E., \& Mueller, H. H. (1998). Fibromyalgia: A retrospective study of 252 consecutive referrals. Canadian Journal of Clinical Medicine, 5(6), 116-127.

Fisher, W., Piazza, C. C., Bowman, L. G., Hagopian, L. P., Owens, J. C., \& Slevin, I. (1992). A comparison of two approaches for identifying reinforcers for persons with severe and profound disabilities. Journal of Applied Behavior Analysis, 25(2), 491-498. https://doi.org/10.1901/jaba.1992.25-491

Gracefire, P. (2016). Introduction to the Concepts and Clinical Applications of Multivariate Live Z-Score Training, PZOK and sLORETA FEEDBACK. In T. Collura \& J. A. Frederick (Eds.), Handbook of Clinical QEEG and Neurofeedback (pp. 326383). Routledge.

Hammer, B. U., Colbert, A. P., Brown, K. A., \& Ilioi, E. C. (2011). Neurofeedback for Insomnia: A Pilot Study of Z-Score SMR and Individualized Protocols. Applied Psychophysiology and Biofeedback, 36(4), 251-264. https://doi.org/10.1007/s10484$\underline{011-9165-y}$ 
Howe, R. C., \& Sterman, M. B. (1972). Cortical-subcortical EEG correlates of suppressed motor behavior during sleep and waking in the cat. Electroencephalography and Clinical Neurophysiology, 32(6), 681-695.

https://doi.org/10.1016/0013-4694(72)90104-6

INFOCOP: Consejo General de la Psicología de España. (2006). ¿QUÉ MIDE REALMENTE EL SCL-90-R?

http://www.infocop.es/view article.asp?id=673\#inicio

Kanda, M., Nagamine, T., Ikeda, A., Ohara, S., Kunieda, T., Fujiwara, N., Yazawa, S., Sawamoto, N., Matsumoto, R., Taki, W., \& Shibasaki, H. (2000). Primary somatosensory cortex is actively involved in pain processing in human. Brain Research, 853(2), 282-289. https://doi.org/10.1016/S0006-8993(99)02274-X

Kayiran, S., Dursun, E., Ermutlu, N., Dursun, N., \& Karamürsel, S. (2007). Neurofeedback in fibromyalgia syndrome. The Journal of the Turkish Society of Algology, 19(3), 47-53.

Kayıran, S., Dursun, E., Dursun, N., Ermutlu, N., \& Karamürsel, S. (2010). Neurofeedback Intervention in Fibromyalgia Syndrome; a Randomized, Controlled, Rater Blind Clinical Trial. Applied Psychophysiology and Biofeedback, 35(4), 293302. https://doi.org/10.1007/s10484-010-9135-9

Kim, J., Loggia, M. L., Cahalan, C. M., Harris, R. E., Beissner, F., Garcia, R. G., Kim, H., Barbieri, R., Wasan, A. D., Edwards, R. R., \& Napadow, V. (2015). The somatosensory link in fibromyalgia: functional connectivity of the primary somatosensory cortex is altered by sustained pain and is associated with clinical/autonomic dysfunction. Arthritis \& Rheumatology, 67(5), 1395-1405. https://doi.org/10.1002/art.39043

Michel, C. M., \& Brunet, D. (2019). EEG sourceimaging: a practical review of the analysis steps. Frontiers in Neurology, 10, 325. https://doi.org/10.3389/fneur.2019.00325

Minhoto, G., Roizenblatt, S., \& Tufik, S. (1997). The effect of biofeedback in fibromyalgia. Sleep Research, $26,573$.

Mueller, H. H., Donaldson, C. C., Nelson, D. V., \& Layman, M. (2001). Treatment of fibromyalgia incorporating EEG-Driven stimulation: A clinical outcomes study. Journal of Clinical Psychology, 57(7), 933-952.

National Fibromyalgia Association. (2018). Treatment. National Fibromyalgia Association (NFA). http://www.fmaware.org/about-fibromyalgia/treatment/

NIH: Instituto Nacional de Artritis y Enfermedades Musculoesqueléticas y de la Piel. (2018). Fibromialgia. https://www.niams.nih.gov/es/informacion-de-salud/fibromialgia

Ozgocmen, S., Yoldas, T., Kamanli, A., Yildizhan, H., Yigiter, R., \& Ardicoglu, O. (2003). Auditory P300 event related potentials and serotonin reuptake inhibitor treatment in patients with fibromyalgia. Annals of the Rheumatic Diseases, 62(6), 551-555. https://doi.org/10.1136/ard.62.6.551

Sanz, J. (2019, January 26). Proyecto de Apoyo a la Evaluación Psicológica Clínica. https://docplayer.es/40905786-Autoresaaron-t-beck-norman-epstein-gary-brown-y-robert-a-steer.html

Schwartz, M. S. (1995). Biofeedback: A practitioner's guide. In Fibromyalgia syndrome (M. S. Schwartz, pp. 803-819). Guilford Press.

Smith, M. L. (2017). sLORETA in clinical practice: not all ROls are created equal. In T. F. Collura \& J. A. Frederick (Eds.), Handbook of clinical QEEG and neurotherapy (pp. 283-299). Routledge, Taylor \& Francis Group.

Soutar, R. G., \& Longo, R. E. (2011). Doing neurofeedback: An introduction. ISNR Research Foundation. 